\title{
A Duodenal Ulcer Caused by Pancreatic Ductal Hypertension with Chronic Pancreatitis
}

\author{
Akira Imoto ${ }^{1}$, Daisuke Masuda ${ }^{1}$, Atsushi Okuda ${ }^{1}$, Wataru Takagi ${ }^{1}$, Saori Onda ${ }^{1}$, \\ Tatsushi Sano ${ }^{1}$, Takashi Mikami ${ }^{1}$, Malak Mohamed ${ }^{1,2}$, Takeshi Ogura ${ }^{1}$ and Kazuhide Higuchi ${ }^{1}$
}

\begin{abstract}
We herein describe the case of a 67-year-old woman with a duodenal ulcer thought to be caused by elevated pancreatic ductal pressure. The patient complained of continuous upper abdominal pain. Her medical history included idiopathic chronic pancreatitis. Endoscopy revealed a huge duodenal ulcer located on the inferior duodenal angle, which had not been seen on endoscopic retrograde pancreatography two months previously. A combination study using endoscopy and contrast imaging confirmed the relationship between the duodenal ulcer and the pancreatic branch duct. To our knowledge, this is the first case of duodenal ulcer thought to be caused by elevated pancreatic ductal pressure.
\end{abstract}

Key words: duodenal ulcer, chronic pancreatitis, pancreatic ductal hypertension, pancreatic stone

(Intern Med 54: 3151-3155, 2015)

(DOI: 10.2169/internalmedicine.54.5779)

\section{Introduction}

A duodenal ulcer is one of the most commonly encountered gastrointestinal pathologies. Helicobacter pylori $(H P)$ infection and the consumption of nonsteroidal antiinflammatory drugs (NSAIDs) are the two major causes of this condition $(1,2)$. Approximately $95 \%$ of duodenal ulcers occur in the duodenal bulb or within $5 \mathrm{~cm}$ of the pyloric ring. Almost all of the other 5\% form between the bulb and the ampulla. Duodenal ulcers occurring at the anal side rather than at the ampulla of Vater are rare $(3,4)$. Chronic pancreatitis (CP) is an irreversible and progressive inflammatory process, featuring pathological modifications of fibrosis, inflammatory infiltration, and the destruction of exocrine and endocrine tissue $(5,6)$. As a result, there are specific morphological changes in the parenchyma and pancreatic ducts, causing intraductal obstruction and ductal hypertension of the pancreas $(7,8)$. Although these conditions sometimes lead to the development of pancreatic stones, pseudocysts, common bile duct stenosis and so on, duodenal ulcers associated with $\mathrm{CP}$ have been very rarely reported worldwide. We herein report a case of duodenal ulcer apparently induced by elevation of the pancreatic ductal pressure in a patient with $\mathrm{CP}$.

\section{Case Report}

A 67-year-old woman was first admitted to our hospital to undergo treatment for CP three years ago. She did not have a drinking habit, but was a smoker. She had no family history of gastrointestinal disease. Many pancreatic stones were found in the pancreas parenchyma and within the pancreatic ducts. The pancreatic stones were treated with extracorporeal shock wave lithotripsy (ESWL) and endoscopic retrograde pancreatography (ERP), but few of the stones were removed because of stenosis and bending of the main pancreatic duct (MPD). The stenotic portions were dilated with a balloon and a catheter, followed by placement of a 7-French (Fr) plastic stent, which was exchanged every three months. At the fifth stent exchange, the plastic stent was removed because the stenosis of the pancreas head and the dilation downstream were judged to be relatively improved.

Three weeks later, the patient experienced upper quadrant pain and was hospitalized. Multiple protease inhibitors and a histamine H2-receptor antagonist were administered for a long period. In addition, the patient had been taking loxoprofen sodium for low back pain for more than ten years.

${ }^{1}$ Second Department of Internal Medicine, Osaka Medical College, Japan and ${ }^{2}$ Department of Gastroenterology, Sohag University, Egypt Received for publication May 15, 2015; Accepted for publication June 10, 2015

Correspondence to Dr. Akira Imoto, akiraimoto27@gmail.com 

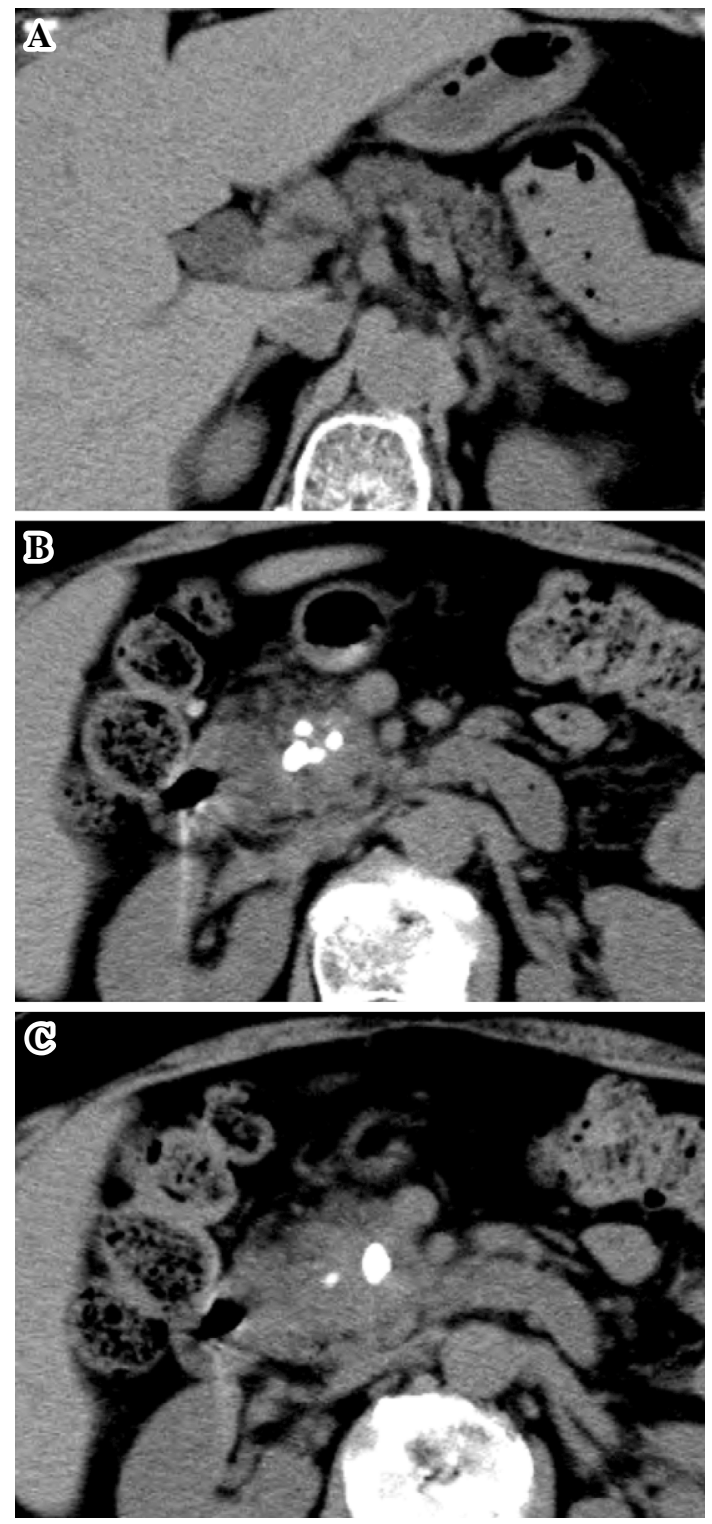

Figure 1. No evident acute pancreatitis was seen on computed tomography (CT) performed on the day of admission. The pancreas as a whole was atrophied (A). Many pancreatic stones were observed within the main pancreatic duct (MPD) (B), the parenchyma, and the branch $\operatorname{duct}(\mathrm{C})$.

On examination, her temperature was $36.1^{\circ} \mathrm{C}$, heart rate was 80 beats per minute, blood pressure was $129 / 68 \mathrm{mmHg}$, and her respiratory rate was 16 breaths per minute. Spontaneous and pressure pain were observed in the epigastric region. The laboratory test results included leukocytosis $\left(9.93 \times 10^{3} /\right.$ $\mu \mathrm{L}$, neutrophils $70.8 \%$ ) with an elevated C-reactive protein level $(9.90 \mathrm{mg} / \mathrm{dL})$. The pancreatic enzyme levels were not elevated. She was negative for anti- $H P$ antibodies. The serum gastrin level was normal.

Based on a computed tomography (CT) study performed on the day of admission, there were no evident changes in the patient's acute pancreatitis (Fig. 1). The pancreatic stones had not changed in size or position compared with the observations of the CT scans taken two months earlier. No stones or sludge were observed within the bile ducts.
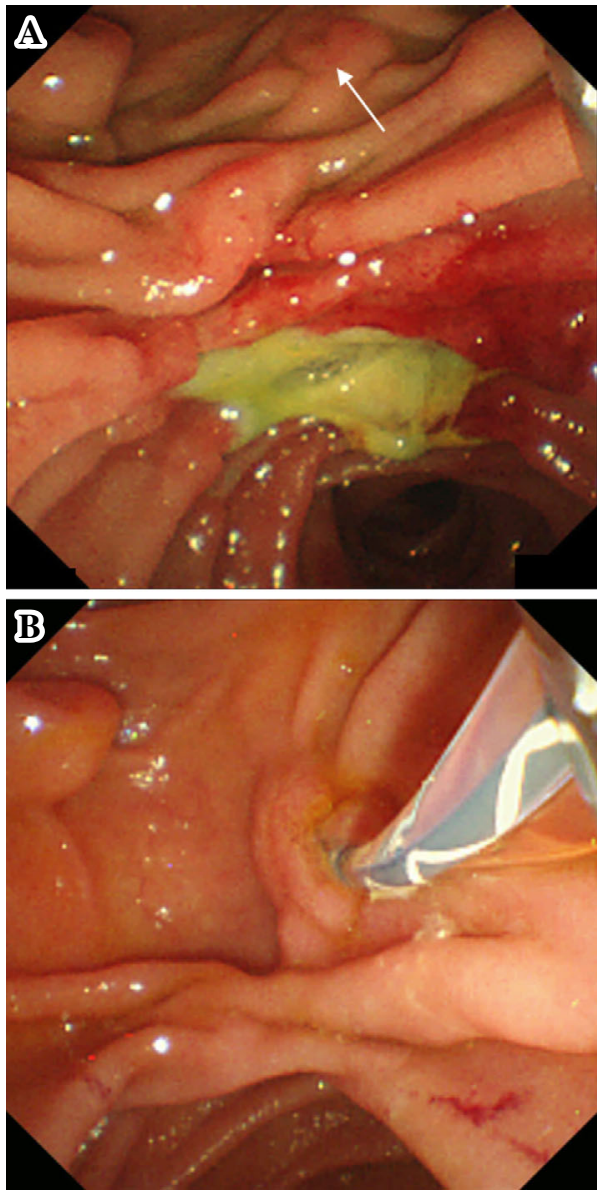

Figure 2. Duodenoscopy using a side-viewing endoscope revealed a large duodenal ulcer with thick fur on the inferior duodenal angle (A), which had not been seen on endoscopic retrograde pancreatography (ERP) two months previously (B). The white arrow indicates Vater's papilla.

The cause of the patient's abdominal pain was unknown. She was managed by fasting. On the third day, esophagogastroduodenoscopy (EGD) revealed a large duodenal ulcer with thick fur on the inferior duodenal angle (Fig. 2A), which had not been seen on ERP two months prior (Fig. 2B) and was compatible with the cause of the patient's abdominal pain. The loxoprofen was discontinued and a proton pump inhibitor (PPI) was added to her usual drug regimen. A histological examination of the ulcer excluded neoplasms and chronic inflammatory diseases. ERP was performed six days after the EGD. Fasting with PPI administration improved the duodenal ulcer. After an injection of contrast media into the MPD, the dilated branch duct filled with pancreatic stones was observed (Fig. 3A), with results consistent with those observed two months prior (Fig. 3B). Delicate manipulation of the guide wire into the branch duct revealed a fistula between the branch duct and the duodenal ulcer (Fig. 3C, D). The fistula was thought to have been induced by hypertension of the branch duct. As the stricture of the pancreas head had caused ductal hypertension downstream, including in the branch duct, a 7-Fr plastic stent was inserted into the MPD. Fortunately, the patient's status 

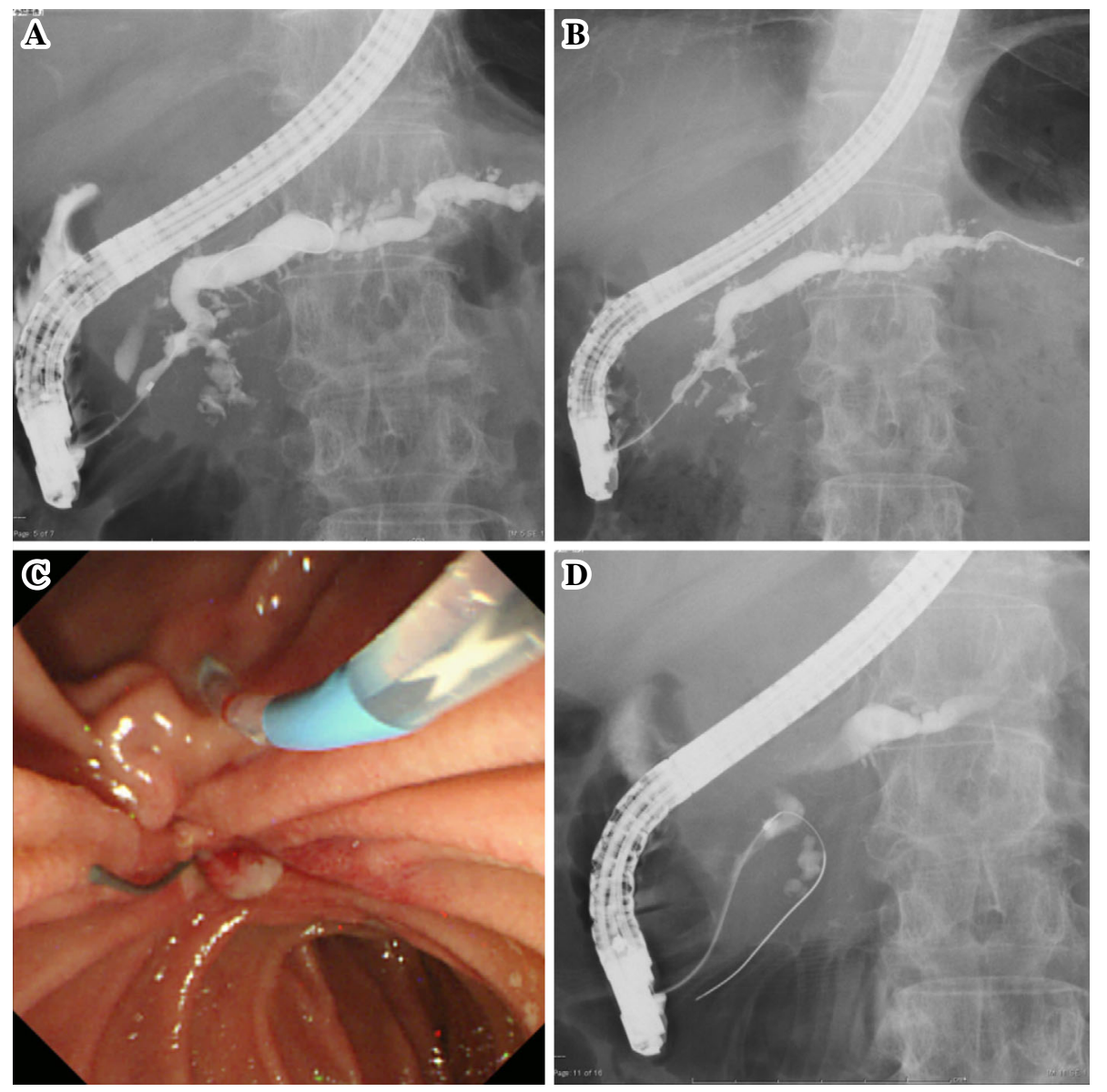

Figure 3. The duodenal ulcer was improved by fasting and the administration of a proton pump inhibitor (PPI) and a protease inhibitor (C), compared with the first-look esophagogastroduodenoscopy (EGD). Endoscopic retrograde pancreatography (ERP) disclosed the irregularly dilated main pancreatic duct (MPD) and branch duct filled with multiple stones (A), which were the same findings as had been noted two months prior $(B)$. Delicate manipulation of the guide wire into the branch duct revealed a fistula between the branch duct and the duodenal ulcer (C, D).

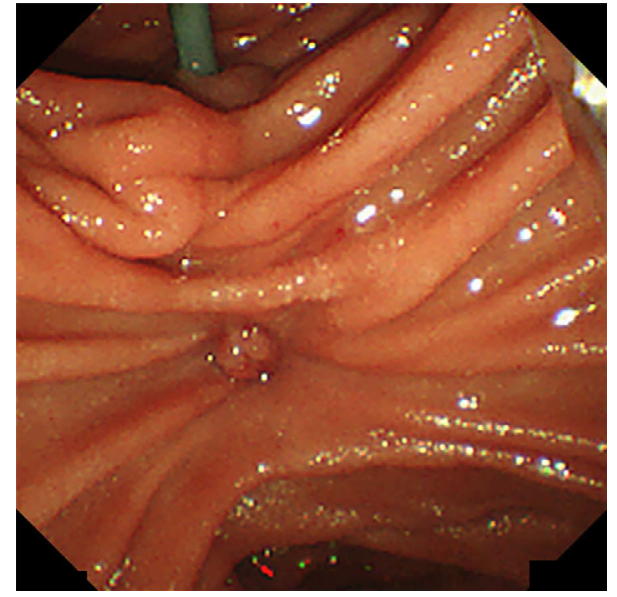

Figure 4. Esophagogastroduodenoscopy (EGD) performed one month after stenting showed further improvement of the duodenal ulcer.

gradually improved. After discharge from the hospital, the patient has remained symptom-free, with her duodenal ulcer improving at present (Fig. 4, 1 month after stenting).

Discussion

Many studies have shown that $H P$ plays a role in the multifactorial etiology of peptic ulcer disease, and there is an interplay of many factors, such as acid attack and the mucosal defense (9-11). The prevalence of $H P$ infection in patients with duodenal ulcers has consistently been found to be between $90-100 \%(12,13)$; therefore, eradication of $H P$ is now the mainstay of treatment for peptic ulcer disease. In the present case, the patient was negative for anti-HP antibodies. Representative causes of $H P$-negative duodenal ulcers are NSAIDs or other ulcerogenic drugs, smoking, older age, gastric hypersecretion (Zollinger-Ellison syndrome), diseases of the duodenal mucosa (Crohn's disease, neoplasms/lymphoma, infections), and concomitant diseases (malignancy, chronic renal failure, liver cirrhosis) (14). Although the present patient was a smoker, was relatively older and had been taking NSAIDs, the presence of a fistula between the branch duct and the duodenum strongly suggested that the ulcer was related to a pancreatic abnormality. 
Duodenal ulcers have been reported to be able to cause choledoco-duodenal or pancreato-duodenal fistulas (15-17); however, there have been no reports of pancreato-duodenal fistulas or duodenal ulcers induced by pancreatic abnormalities. Sung et al. reported that pancreatic juice contributed to recurrent peptic ulcers (18). An animal study has shown that esophageal mucosal injury can be caused by external influx of bile and pancreatic secretions (19). Thus, the leakage of pancreatic juice thorough the fistula was likely the cause of the duodenal ulcer in the present case. The unusual location of the ulcer and the episode of stent retrieval before she suffered abdominal pain supported this idea.

Although contrast radiography of the branch duct did not show any results significantly different from those obtained during the previous ERP, the pressure in the branch duct was probably elevated. The reason for the branch duct hypertension was thought to be the remaining stricture of the MPD at the proximal side, rather than the branch site. Therefore, dilation of the MPD stricture of the pancreas head by stenting was selected as the treatment for this patient. The patient's good clinical course after stenting was compatible with our hypothesis.

Strictures of the MPD are seen in about half of patients with CP. The strictures are usually located in the pancreas head, and are caused by inflammation or fibrosis (6). If the stenosis can be overcome, the MPD can be decompressed and the pain can be relieved $(20,21)$. In the present case, the stricture of the MPD had been dilated with a 7-Fr plastic stent until three weeks before the abdominal pain occurred. Based on our hypothesis, the dilation of the stricture was not sufficient, and the stent should have been left in place. The European Society of Gastroenterology (ESGE) recommends treating dominant MPD stricture by inserting a single 10 -Fr plastic stent (22). Dilation by a 10 -Fr stent instead of a 7-Fr stent might prevent the development of this type of duodenal ulcer. Recent studies performed with specially made auto-expandable metallic stents have shown a partial improvement in the patients' pain after stent placement $(23,24)$. Covered metallic stenting is another possible method that might have been useful for treating stenosis of the MPD in the present case.

It is unknown whether gastric acid or pancreatic enzymes were related to the development of the ulcer in the present patient. Fasting with the administration of a PPI was added to the administration of a protease inhibitor, and this markedly improved the patient's duodenal ulcer. Fasting may have decreased the ductal hypertension by reducing the amount of pancreatic juice excreted. The PPI or protease inhibitor may have contributed to the improvement of the patient's ulcer by blocking gastric acid or pancreatic enzyme production.

We encountered a case of duodenal ulcer thought to be induced by pancreatic ductal hypertension. A combination study using endoscopy and contrast imaging was useful for making an accurate diagnosis. Dilation of the stenosis of the pancreatic head using a plastic stent was sufficient to enable the patient to successfully recover.

The authors state that they have no Conflict of Interest (COI).

\section{Acknowledgement}

We thank Dr. Etsuko Makino, Setsuko Tsujimura, Mayumi Hagiwara, and Natsuki Imoto for their helpful suggestions regarding the manuscript.

\section{References}

1. Wolfe MM, Lichtenstein DR, Singh G. Gastrointestinal toxicity of nonsteroidal antiinflammatory drugs. N Engl J Med 340: 18881899, 1999.

2. Huang JQ, Sridhar S, Hunt RH. Role of Helicobacter pylori infection and non-steroidal anti-inflammatory drugs in peptic-ulcer disease: a meta-analysis. Lancet 359: 14-22, 2002.

3. Rodriguez HP, Aston JK, Richardson CT. Ulcers in the descending duodenum. Postbulbar ulcers. Am J Roentgenol Radium Ther Nucl Med 119: 316-322, 1973.

4. Al-Bahrani ZR, Kassir ZA, Al-Doree W. The location and multiplicity of chronic duodenal ulcer (A study of 1320 patients in Iraq). Gastroenterol Jpn 15: 539-542, 1980.

5. Bornman PC, Beckingham IJ. ABC of diseases of liver, pancreas, and biliary system. Pancreatic tumours. BMJ 322: 721-723, 2001.

6. Seicean A, Vultur S. Endoscopic therapy in chronic pancreatitis: current perspectives. Clin Exp Gastroenterol 8: 1-11, 2014.

7. Bradley EL 3rd. Pancreatic duct pressure in chronic pancreatitis. Am J Surg 144: 313-316, 1982.

8. Karanjia ND, Singh SM, Widdison AL, Lutrin FJ, Reber HA. Pancreatic ductal and interstitial pressures in cats with chronic pancreatitis. Dig Dis Sci 37: 268-273, 1992.

9. Levi S, Beardshall K, Haddad G, Playford R, Ghosh P, Calam J. Campylobacter pylori and duodenal ulcers: the gastrin link. Lancet 1: 1167-1168, 1989.

10. Peura DA. Ulcerogenesis: integrating the roles of Helicobacter pylori and acid secretion in duodenal ulcer. Am J Gastroenterol 92: 8S-13S; discussion S-6S, 1997.

11. Tytgat GN, Noach LA, Rauws EA. Helicobacter pylori infection and duodenal ulcer disease. Gastroenterol Clin North Am 22: 127139, 1993.

12. Prasad S, Mathan M, Chandy G, et al. Prevalence of Helicobacter pylori in southern Indian controls and patients with gastroduodenal disease. J Gastroenterol Hepatol 9: 501-506, 1994.

13. Goh KL. Prevalence of and risk factors for Helicobacter pylori infection in a multi-racial dyspeptic Malaysian population undergoing endoscopy. J Gastroenterol Hepatol 12: S29-S35, 1997.

14. Gisbert JP, Calvet X. Review article: Helicobacter pylori-negative duodenal ulcer disease. Aliment Pharmacol Ther 30: 791-815, 2009.

15. Wong WM, Hu WH, Lai KC. Images of interest. Hepatobiliary and pancreatic: choledochoduodenal fistula secondary to duodenal ulcer disease. J Gastroenterol Hepatol 19: 829, 2004.

16. Meiselman MS, Agha FP. Fistula to the pancreatic duct from a peptic ulcer. Case report and literature review. J Clin Gastroenterol 10: $537-540,1988$.

17. Merrill JR. Fistulation to the pancreatic duct complicating duodenal peptic ulcer. Gastroenterology 87: 957-959, 1984.

18. Sung HY, Kim JI, Park YB, et al. The papilla of Vater just below the pylorus presenting as recurrent duodenal ulcer bleeding. Intern Med 46: 1853-1856, 2007.

19. Cross FS, Wangensteen $\mathrm{OH}$. Role of bile and pancreatic juice in production of esophageal erosions and anemia. Proc Soc Exp Biol Med 77: 862-866, 1951.

20. Seven G, Schreiner MA, Ross AS, et al. Long-term outcomes as- 
sociated with pancreatic extracorporeal shock wave lithotripsy for chronic calcific pancreatitis. Gastrointest Endosc 75: 997-1004.e1, 2012.

21. Guda NM, Partington S, Freeman ML. Extracorporeal shock wave lithotripsy in the management of chronic calcific pancreatitis: a meta-analysis. JOP: Journal of the pancreas 6: 6-12, 2005.

22. Dumonceau JM, Delhaye M, Tringali A, et al. Endoscopic treatment of chronic pancreatitis: European Society of Gastrointestinal Endoscopy (ESGE) Clinical Guideline. Endoscopy 44: 784-800, 2012.
23. Sauer B, Talreja J, Ellen K, Ku J, Shami VM, Kahaleh M. Temporary placement of a fully covered self-expandable metal stent in the pancreatic duct for management of symptomatic refractory chronic pancreatitis: preliminary data (with videos). Gastroint Endosc 68: 1173-1178, 2008.

24. Park do H, Kim MH, Moon SH, Lee SS, Seo DW, Lee SK. Feasibility and safety of placement of a newly designed, fully covered self-expandable metal stent for refractory benign pancreatic ductal strictures: a pilot study (with video). Gastrointest Endosc 68: 1182-1189, 2008.

(C) 2015 The Japanese Society of Internal Medicine http://www.naika.or.jp/imonline/index.html 